\title{
DESEMPENHO DE UM PULVERIZADOR PNEUMÁTICO COSTAL MOTORIZADO SOB DIFERENTES CONDIÇÕES ${ }^{1}$
}

\section{CASIMIRO D. GADANHA JÚNIOR ${ }^{2}$, MARCO A. F. DA COSTA ${ }^{3}$, MARCOS MILAN ${ }^{4}$}

RESUMO: O objetivo do trabalho foi avaliar a uniformidade de vazão de um pulverizador pneumático costal motorizado, utilizado no controle do mosquito vetor da dengue, sob várias condições operacionais de aplicação. $O$ experimento foi planejado como um delineamento inteiramente casualizado, no esquema fatorial 2 × 7, com cinco repetições, sendo os fatores de variação dois diâmetros de orifício do disco dosador e sete ângulos de aplicação em relação ao plano horizontal. Foi avaliado também o efeito das pressões sobre a vazão por meio dos testes de Pearson. A interação dos fatores diâmetro do orifício do disco dosador e ângulo de aplicação foi significativa para a vazão. $\mathrm{O}$ disco dosador com diâmetro de $0,42 \mathrm{~mm}$ apresentou maior uniformidade entre as vazões médias, variando de 12,8 a $38,8 \mathrm{~mL} \mathrm{~min}^{-1}$, para o intervalo de inclinação do ducto aplicador de $60^{\circ}$ a $-60^{\circ}$ e apresentou correlação linear em função do ângulo, com coeficiente de determinação igual a 0,9861 . Houve uma expressiva correlação entre a vazão média e a pressão determinada no ponto $\mathrm{P} 3$, próximo ao bocal, com valores do coeficiente de correlação de 0,988 para o disco A e de 0,985 para o disco B. $\mathrm{O}$ disco de menor diâmetro deve ser utilizado. Na troca do disco, o operador deve ser orientado e treinado para corrigir o tempo de aplicação em função das diversas situações na área urbana.

PALAVRAS-CHAVE: Aedes aegypti, controle químico, pulverizador.

\section{PERFORMANCE OF THE MOTORIZED KNAPSACK MISTBLOWER UNDER DIFFERENT OPERATIONAL CONDITIONS}

SUMMARY: This study aims to evaluate the outflow uniformity of knapsack mist-blowers, powered by two stroke internal combustion engine for the control of the mosquito vector of dengue (Aedes aegypti) under different operational conditions. The experiment was planned as a completely randomized factorial $2 \times 7$ with five repetitions being the variation factors two diameters of orifices of the disc dosimeter and seven application angles in relation to the horizontal plan. It was also evaluated the pressure effect on the flow rate through the Pearson tests. The results showed significant association between the factors diameter of the dosimeter and the application angles for the flow rate. The dosimeter disc of diameter $0.42 \mathrm{~mm}$ presented greater uniformity between the average flow rate (12.8 to $\left.38.8 \mathrm{~mL} \mathrm{~min}^{-1}\right)$ for the band of angles used $\left(60^{\circ}\right.$ to $\left.-60^{\circ}\right)$ with determination coefficient up to 0.9861 . It had a higher correlation between the average flow rate and the pressure determined at the point P3 close to the nozzle, with a correlation coefficient of 0.988 for disc A and 0.985 for disc B. The disc of smaller diameter must be used. The operator will have to be guided and trained for the choice of discs and correction of the time of application in the diverse situations of field in the urban area.

KEYWORDS: Aedes aegypti, chemical control, sprayer.

\footnotetext{
${ }^{1}$ Extraído da dissertação de mestrado do segundo autor.

${ }^{2}$ Eng $^{0}$ Agrônomo, Prof. Dr., Departamento de Engenharia Rural, ESALQ/USP, Piracicaba - SP, e-mail: cdgadanh@esalq.usp.br

${ }^{3}$ Eng ${ }^{0}$ Agrônomo, Superintendência de Controle de Endemias, Secretaria de Saúde do Estado de São Paulo, São Paulo - SP, e-mail: somarcamp@uol.com.br

${ }^{4}$ Eng ${ }^{\mathrm{o}}$ Agrônomo, Prof. Dr., Departamento de Engenharia Rural, ESALQ/USP, Piracicaba - SP.

Recebido pelo Conselho Editorial em: 12-3-2003

Aprovado pelo Conselho Editorial em: 26-7-2004
} 


\section{INTRODUÇÃO}

Dentre os mosquitos associados ao meio urbano, o Aedes aegypti, principal transmissor da dengue, é uma das espécies mais perigosas em áreas tropicais. O mosquito da dengue está disperso em todos os Estados do Brasil, sendo confirmados cerca de 238 mil casos dessa doença no ano de 2000, segundo a Fundação Nacional de Saúde - FUNASA (2001). No Estado de São Paulo, até setembro de 2001, o número de casos confirmados da dengue foi de 51.115, contra os 3.529 casos no ano anterior, representando um aumento de 1.448,4\%. O número de municípios infestados nesse Estado pelo $A$. aegypti passou de 126 em 1987, para 482 em 2001, representando um aumento de 382,5\%, segundo o Centro de Vigilância Epidemiológica - CVE (2001).

Para o controle dessa espécie de mosquito ou vetor, são utilizadas medidas preventivas, como o saneamento básico, e medidas emergenciais ou de caráter temporário, como o uso de agrotóxicos e o controle biológico. No Brasil, o saneamento básico não é prioridade no âmbito das políticas públicas. Segundo dados da Fundação Instituto Brasileiro de Geografia e Estatística - FIBGE (2001), apenas 451 municípios fazem coleta seletiva de lixo e 352 realizam reciclagem. O controle biológico apresenta problemas operacionais, como a produção em pequena escala de produtos desse tipo e a falta de metodologias adequadas para a aplicação dos mesmos, tornando-o insustentável em grandes epidemias. O controle químico ainda é o mais importante no controle integrado de mosquitos vetores de doenças para a eliminação da forma adulta (alada) do vetor.

Os equipamentos mais comumente usados na saúde pública para a aplicação dos agrotóxicos são o pulverizador a pressão com jato lançado e manual, o pneumático costal motorizado, o pneumático montado em base móvel, o termonebulizador manual ou montado em base móvel, a polvilhadora manual e o equipamento de aplicação aérea.

A tecnologia de aplicação mais utilizada para o controle da forma alada do mosquito A. aegypti é a espacial, com gotas no tamanho de aerossol e que visa a manter as partículas líquidas suspensas o tempo suficiente para atingir os insetos que estão no espaço tratado (ABNT, 1988; ASAE, 1996).

Os equipamentos de pulverização aérea e os pneumáticos montados em bases móveis, que geram partículas no tamanho de aerossol, não são recomendados no Estado de São Paulo para o controle do mosquito vetor da dengue. Nessas aplicações, a interação entre diversos fatores (principalmente climáticos) afetam a trajetória das partículas, dificultando a penetração no espaço interno das residências onde as fêmeas adultas do mosquito habitualmente estão presentes. Em função dessas restrições, a Superintendência de Controle de Endemias - SUCEN, órgão responsável pelo controle da dengue no Estado de São Paulo, utiliza, em praticamente 100\% das aplicações de inseticidas para o controle do mosquito, o pulverizador pneumático costal motorizado. Esse tipo de pulverizador é constituído de um motor de combustão interna monocilíndrico de ciclo dois tempos, à gasolina, de baixa potência, que trabalha na rotação máxima acionando um ventilador radial. O líquido contido no reservatório de calda pode ser conduzido por gravidade ou por uma bomba centrífuga, por meio de um sistema de mangueiras, passando por um registro de saída de calda até o mecanismo dosador instalado em ducto alongado responsável pela condução da corrente de ar e melhor posicionamento da pulverização com relação ao alvo. Possui, ainda, o registro e o bico (ABNT, 1993). O pulverizador pneumático costal motorizado, apesar de ser muito utilizado em saúde pública, no Estado de São Paulo, apresenta apenas três fabricantes no Brasil, ofertando no máximo oito modelos. JOLLANDS (1991), utilizando óxido cuproso em pulverizações para controle de Hemileia vastatrix em café, avaliou o desempenho de seis pulverizadores pneumáticos costais motorizados com relação à variação de vazão, em três posições de aplicação, $45^{\circ} ; 0^{\circ}$ e $-20^{\circ}$ em relação ao plano horizontal. Das seis máquinas avaliadas, cinco apresentaram grandes variações na vazão de 100 até 365\%, quando a posição foi mudada. 
Constata-se, empiricamente, que a aplicação pode mostrar-se ineficiente em função da forma de utilização do pulverizador e, no entanto, a bibliografia de saúde pública no Brasil é carente de dados técnicos de desempenho operacional dessas máquinas. $\mathrm{O}$ trabalho teve como objetivo avaliar a uniformidade de vazão de um pulverizador pneumático costal motorizado, disponível no mercado nacional sob o efeito de diferentes ângulos de aplicação, diâmetros de disco dosador e pressões.

\section{MATERIAL E MÉTODOS}

O trabalho foi realizado no Laboratório de Tecnologia de Aplicação de Defensivos Agrícolas da Escola Superior de Agricultura “Luiz de Queiroz”, Universidade de São Paulo, em Piracicaba - SP.

Utilizou-se um pulverizador pneumático costal motorizado, modelo MULTISPRAY 2000 LD, série 9700, com motor de combustão interna, ciclo dois tempos, modelo JET 400, com potência de $1,2 \mathrm{~kW}(1,6 \mathrm{cv})$. O pulverizador foi fixado em estrutura de apoio, adaptando-se a ela um suporte de madeira para a sustentação do ducto aplicador e um transferidor para a fixação dos ângulos de aplicação em $0^{\circ} ; 20^{\circ} ; 40^{\circ} ; 60^{\circ} ;-20^{\circ} ;-40^{\circ}$ e $-60^{\circ}$ em relação ao plano horizontal.

O disco dosador, que é responsável pela restrição do fluxo de calda no bico é oferecido pelo fabricante em 12 cores diferentes, cada uma delas representada por uma vazão. Na aquisição do equipamento pela SUCEN, o disco bege acompanha a máquina e aparece ocasionalmente com dois diâmetros, cujos valores não são informados. A determinação dos diâmetros dos orifícios foi feita com o auxílio de um projetor óptico, modelo HB 400, e designados posteriormente como disco dosador A para o diâmetro de $0,76 \mathrm{~mm}$ e disco dosador B para o diâmetro de $0,42 \mathrm{~mm}$, sendo o primeiro utilizado pela SUCEN.

A pressão foi medida em quatro pontos do circuito hidráulico e pneumático do pulverizador, utilizando-se de quatro sensores de pressão diferencial, com fundo de escala de $10 \mathrm{kPa}$ e número de ordem MPX5010DP, série MPX. Detalhes do posicionamento dos sensores são apresentados na Figura 1.

Os sensores de pressão foram instalados nos pontos: P1 distante $6 \mathrm{~cm}$ do reservatório de calda instalado na mangueira responsável pela condução do fluxo de ar gerado pelo ventilador radial; P2, distante $80 \mathrm{~cm}$ do registro de saída da calda na mangueira conectada ao reservatório; P3, distante 8,5 cm do corpo dosador e instalado após o registro da saída de calda, e P4, instalado na parte superior do reservatório, registrando a pressurização no interior do mesmo. Os sensores foram instalados nos pontos com a colocação de uma conexão hidráulica de ferro galvanizado em forma de "T".

Um sistema de aquisição de dados, modelo CR10X (Campbell Scientific, Inc.), com entrada analógica para seis canais diferenciais, frequiência de execução de $64 \mathrm{~Hz}$, resolução $0,33 \mu \mathrm{V}$, fundo de escala de 2,5 V e erro $\pm 0,1 \%$, foi programado com o auxílio do programa PC208 W, versão 2.0, para aquisição e armazenamento dos dados em tempo real a cada segundo e posteriormente transferidos para um computador Pentium $200 \mathrm{MHz}, 32 \mathrm{MB}$ de memória de acesso aleatório - RAM e convertidos em arquivo de planilha de dados do programa Microsoft Excel. Os circuitos eletrônicos dos sensores de pressão e do sistema de leitura óptica ligados ao sistema de aquisição de dados foram protegidos contra danos provocados por solventes ou choques, acondicionado-os numa caixa fixada na parte de trás do transferidor.

O reservatório e o sistema de mangueiras foram completados com água da rede de abastecimento, o ducto aplicador foi posicionado no ângulo desejado e a máquina acionada conforme recomendação do fabricante, mantendo-se o motor na máxima aceleração. Após o acionamento do sistema de aquisição dos dados, o registro de calda foi aberto. Toda vez que o nível do reservatório de combustível atingiu cerca de $25 \%$ da sua capacidade ele foi aberto e reabastecido, evitando a parada do 
motor durante os ensaios. Ao final da saída da calda sob forma de gotas, desligou-se o sistema de aquisição de dados e foram efetuados os cálculos de vazão média $\left(\mathrm{L} \mathrm{min}^{-1}\right)$.

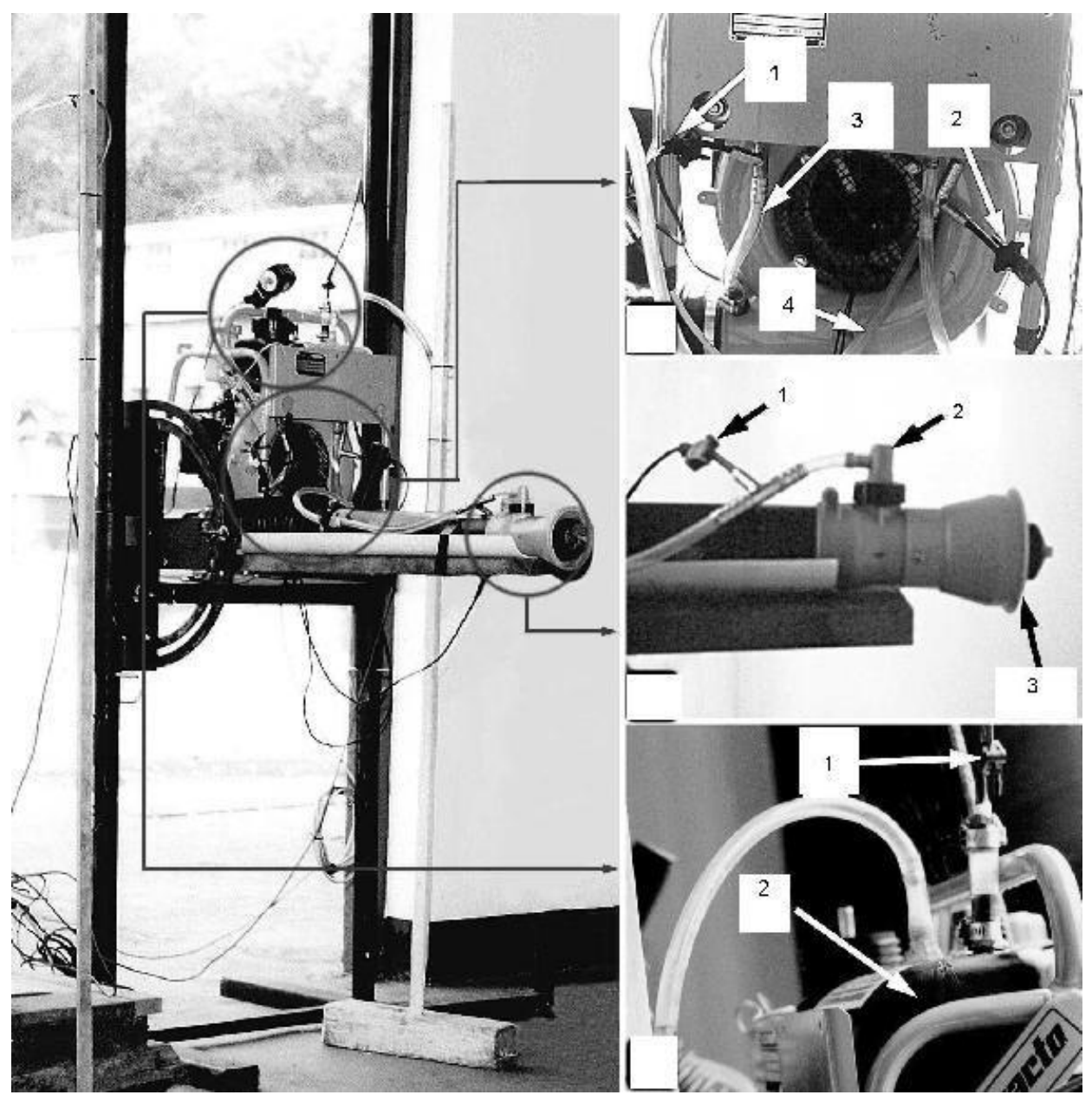

FIGURA 1. Pontos de tomada de pressão e detalhe do sensor no primeiro ponto (P1), segundo (P2), terceiro (P3) e quarto (P4).

O volume total de água consumido no circuito hidráulico não foi coletado durante o ensaio. $\mathrm{O}$ tempo considerado no cálculo da vazão foi obtido após ter cessado totalmente as gotas d'água do pulverizador. A vazão média foi calculada pela eq.(1):

$$
\mathrm{Q}=\frac{\mathrm{V}_{\mathrm{t}}}{\mathrm{t}}
$$

em que,

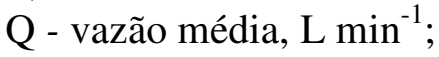

$\mathrm{V}_{\mathrm{t}}$ - volume total consumido, $\mathrm{L}$, e

$\mathrm{t}$ - tempo após ter cessado a saída das gotas, min.

A média geral de vazão $(\bar{Q})$ de cada tratamento é dada pela eq.(2):

$$
\overline{\mathrm{Q}}=\frac{\sum \mathrm{Q}}{\mathrm{n}}
$$

em que, 
$\Sigma$ Q- somatória das vazões das repetições dos tratamentos, e

$\mathrm{n}$ - número de repetições.

O ensaio foi planejado num delineamento inteiramente casualizado, no esquema fatorial $2 \times 7$, com 14 tratamentos e cinco repetições, em que os fatores considerados foram o diâmetro de orifício do disco dosador e os ângulos de inclinação do ducto aplicador.

No estudo dos dados experimentais, utilizou-se o programa computacional Statistical Analisis Systems (SAS) para análise de variância com aplicação do teste F e a comparação entre médias dos tratamentos por meio do teste de Tukey, a 5\% de probabilidade. Para a determinação dos coeficientes de determinação para os discos A e B e as respectivas equações das retas, os ângulos foram convertidos para radianos e, em seguida, utilizou-se o programa Excel. Para verificar a influência das pressões, sobre a vazão média de cada disco dosador, foram feitos os testes de correlação de Pearson e gráficos de tendência.

\section{RESULTADOS E DISCUSSÃO}

$\mathrm{Na}$ análise de variância com aplicação do teste $\mathrm{F}$ e no teste de Tukey, levou-se em consideração um experimento inteiramente casualizado, não balanceado, devido à perda de algumas parcelas. $\mathrm{Na}$ Tabela 1, são apresentados os resultados do teste $\mathrm{F}$ da análise de variância e do teste de Tukey aplicados às médias da interação diâmetro de orifício do disco dosador e ângulo de inclinação do ducto aplicador.

$O$ resultado do teste $\mathrm{F}=645,41$ confirma o efeito significativo $(\mathrm{p}<0,0001)$ da interação ângulo do ducto aplicador e diâmetro de orifício do disco dosador sobre a vazão. Na comparação das médias de vazão $\left(\mathrm{L} \mathrm{min}^{-1}\right)$ dos 14 tratamentos, não foram observadas diferenças significativas entre os tratamentos, cujas médias foram $38,8\left(-60^{\circ} \mathrm{D}_{\mathrm{B}}\right)$ e $36,7 \mathrm{~L} \mathrm{~min}^{-1}\left(-40^{\circ} \mathrm{D}_{\mathrm{B}}\right)$ e entre $36,7\left(-40^{\circ} \mathrm{D}_{\mathrm{B}}\right)$ e $33,9 \mathrm{~L} \mathrm{~min}^{-1}\left(-20^{\circ} \mathrm{D}_{\mathrm{B}}\right)$.

TABELA 1. Teste de Tukey aplicado às médias de vazão $\left(\mathrm{mL} \mathrm{min}^{-1}\right)$ resultante da interação diâmetro de orifício do disco dosador $\left(\mathrm{D}_{\mathrm{A}}\right.$ e $\left.\mathrm{D}_{\mathrm{B}}\right)$ e ângulo de inclinação do ducto aplicador.

\begin{tabular}{|c|c|c|}
\hline \multirow{2}{*}{ Ângulo do Ducto Aplicador $\left({ }^{\circ}\right)$} & \multicolumn{2}{|c|}{ Médias de Vazão $\left(\mathrm{mL} \min ^{-1}\right)$} \\
\hline & $\mathrm{D}_{\mathrm{A}}=0,76 \mathrm{~mm}$ & $\mathrm{D}_{\mathrm{B}}=0,42 \mathrm{~mm}$ \\
\hline$-60^{\circ}$ & $131,6 \mathrm{a}^{1}$ & $38,8 \mathrm{a}$ \\
\hline$-40^{\circ}$ & $124,2 \mathrm{~b}$ & $36,7 \mathrm{ab}$ \\
\hline$-20^{\circ}$ & $111,0 \mathrm{c}$ & $33,9 \mathrm{~b}$ \\
\hline $0^{\circ}$ & $94,8 \mathrm{~d}$ & $27,9 \mathrm{c}$ \\
\hline $20^{\circ}$ & $76,1 \mathrm{e}$ & $23,1 \mathrm{~d}$ \\
\hline $40^{\circ}$ & $55,6 \mathrm{f}$ & $18,4 \mathrm{e}$ \\
\hline $60^{\circ}$ & $42,2 \mathrm{~g}$ & $12,8 \mathrm{f}$ \\
\hline $\mathrm{F}(\mathrm{DxI})=645,41 * *$ & $\begin{array}{l}\text { d.m.s }(5 \%)=2,87 \mathrm{~mL} \mathrm{~min}^{-1} \\
\text { C.V. }=37,7 \% \\
\text { média }=90,8 \mathrm{~mL} \mathrm{~min}^{-1}\end{array}$ & $\begin{array}{l}\text { d.m.s }(5 \%)=3,06 \mathrm{~mL} \mathrm{~min}^{-1} \\
\text { C.V.= }=35,7 \% \\
\text { média }=27,4 \mathrm{~mL} \mathrm{~min}{ }^{-1}\end{array}$ \\
\hline
\end{tabular}

Significativo a $1 \%$ de probabilidade para o teste $\mathrm{F}$.

${ }^{1}$ Médias seguidas de letras minúsculas, diferentes nas colunas, diferem entre si, a 5\% de probabilidade, pelo teste de Tukey.

Comparando-se os diâmetros dos orifícios na Tabela 1 e considerando-se os ângulos da mesma linha, verifica-se que houve aumento médio na vazão de 229,4\%, com o aumento de 0,34 mm no diâmetro. Isso mostra a importância na escolha do disco, podendo ocorrer risco de sub ou superdosagem e perdas significativas de produto. 
Para os discos A e B, as diferenças entre os valores de vazão média para o intervalo de $-60^{\circ}$ e $60^{\circ}$ de inclinação foram, respectivamente, $89,410^{-3} \mathrm{~L} \mathrm{~min}^{-1}$ e $26,010^{-3} \mathrm{~L} \mathrm{~min}^{-1}$. Em termos porcentuais, isso representa diminuição na vazão de 211,8 e $203,1 \%$, respectivamente. As variações porcentuais apresentadas por JOLLANDS (1991) mostram coerência com os dados observados no experimento, atribuindo-se essas variações à pressurização insuficiente no tanque de calda capaz de manter a vazão constante.

O efeito dos diâmetros dos orifícios dos discos restritores e dos ângulos do ducto aplicador sobre a vazão média podem ser vistos na Figura 2.

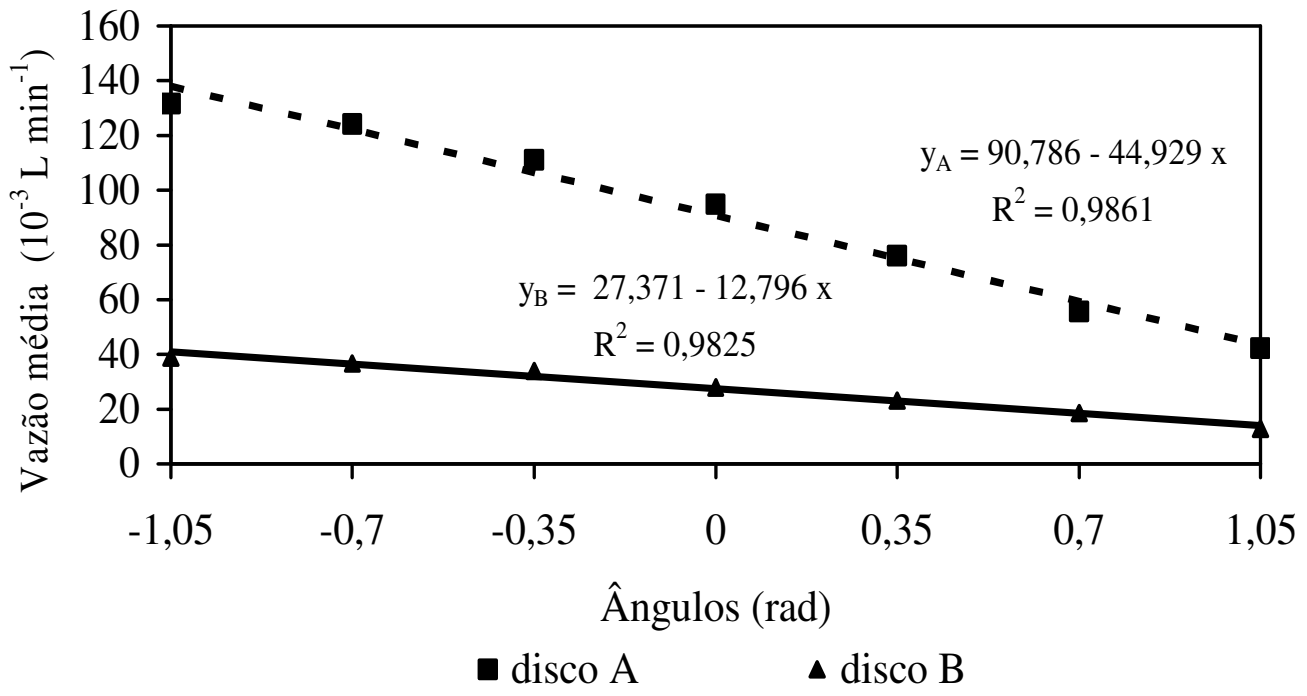

FIGURA 2. Vazões médias nos respectivos tratamentos para os discos dosadores A e B em função do ângulo de aplicação (radianos) do ducto aplicador.

Apesar de as variações serem porcentualmente semelhantes, o disco B (diâmetro $=0,42 \mathrm{~mm}$ ) apresentou maior uniformidade para o intervalo entre $60^{\circ}(-1,05 \mathrm{rad})$ e $-60^{\circ}(1,05 \mathrm{rad})$. Observa-se grande desuniformidade de vazão para as aplicações compreendidas entre os ângulos de $-60^{\circ}$ e $60^{\circ}$ para o disco dosador A (diâmetro $=0,76 \mathrm{~mm}$ ), que é normalmente utilizado pela SUCEN, no controle do Aedes aegypti.

Os coeficientes de determinação $\left(\mathrm{R}^{2}\right)$ foram 0,9861 e 0,9825 para os discos A e B, respectivamente, e os valores de vazão ajustados na reta, por meio da regressão, mostraram que existe relação linear entre as leituras e os ângulos de aplicação.

$\mathrm{Na}$ operacionalização do trabalho em áreas urbanas, habitualmente, a maioria das aplicações ocorrem em portas e janelas de casas térreas e de sobrados onde os ângulos de inclinação do ducto aplicador são positivos em relação ao plano horizontal, daí a maior importância da faixa entre $0^{\circ}$ e $60^{\circ}$. Por outro lado, devido à diversidade de relevos encontrados na área urbana, não se descarta a possibilidade de posicionamento do ducto aplicador em ângulos negativos, cuja desuniformidade de vazão também existe.

Pode-se observar na Figura 3 que, no ponto P3, localizado próximo ao corpo do dosador, ocorre aumento da pressão de serviço com o aumento da vazão para ambos os discos dosadores. As pressões nos pontos P2 e P4, apesar de estarem em pontos distintos, apresentam valores muito semelhantes, considerando a pequena coluna d’água existente dentro do reservatório, correspondente a $0,8 \mathrm{kPa}$ ( $8 \mathrm{~cm}$ de coluna d'água) e as perdas de carga entre os dois pontos. 


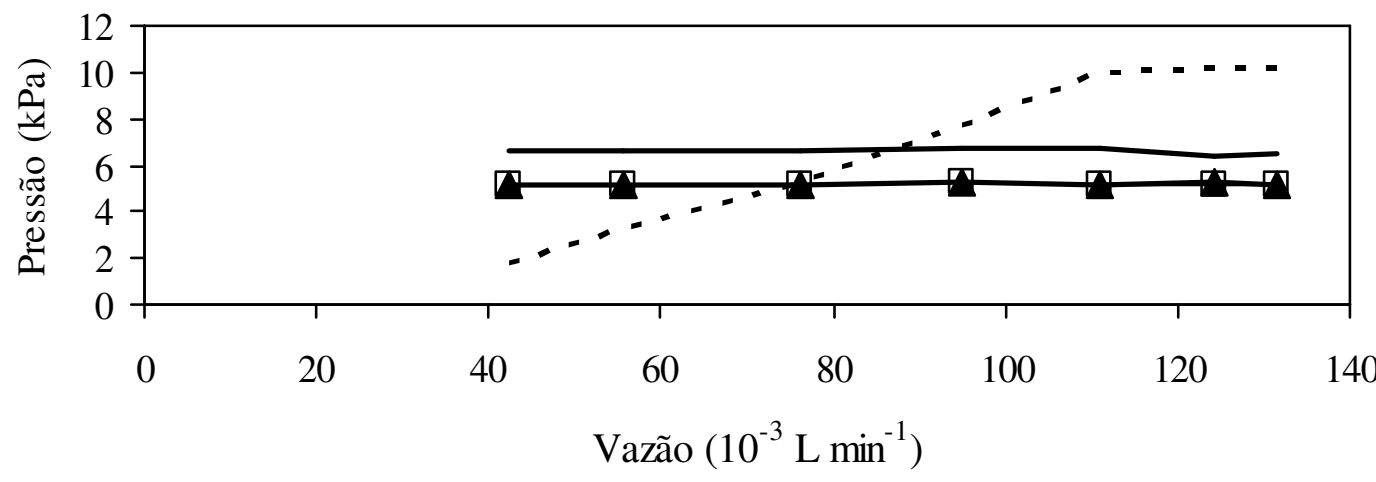

DISCO A

— pressão $1 \longrightarrow$ pressão 2 - - - pressão $3 \longrightarrow$ pressão 4

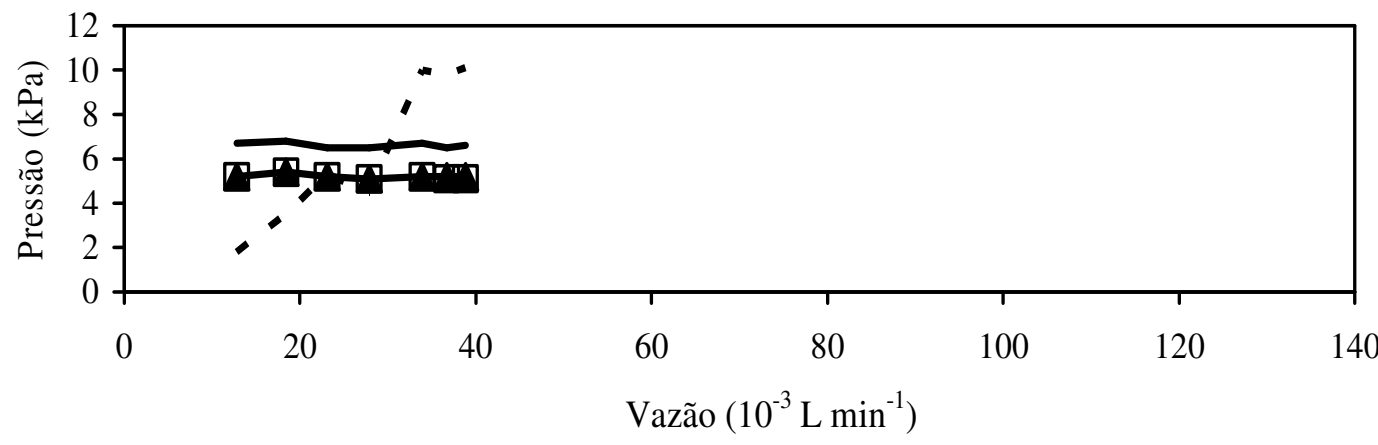

DISCO B

pressão $1 \longrightarrow$ pressão 2 - - - pressão $3 \quad \square$ pressão 4

FIGURA 3. Pressões de serviço P1 (pressão1), P2 (pressão 2), P3 (pressão 3) e P4 (pressão 4) em função das vazões médias para os discos dosadores A e B.

$\mathrm{Na}$ Tabela 2, verifica-se que existe correlação positiva entre as pressões no ponto P3 e as vazões para ambos os discos, com valores de coeficiente de correlação de 0,988 e 0,985, respectivamente; além das evidências estatísticas $(\mathrm{p}=0)$ de que os dados são correlacionados. Ocorre também correlação positiva entre as pressões nos pontos P2 e P4 e as vazões do disco dosador A; no entanto, não existem evidências estatísticas para rejeitar a hipótese de dados correlacionados $(\mathrm{p}>0,05)$. Isto se justifica pelo fato de que as pressões nos pontos P2 e P4 foram apenas monitoradas, mantendo-as constantes.

TABELA 2. Teste de correlação de Pearson para pressões e vazões médias .

\begin{tabular}{cccc}
\hline Discos Dosadores & Pressões de Serviço & Coeficiente de Correlação & $\mathrm{p}$ - valor \\
\hline \multirow{3}{*}{ Disco Dosador A } & P1 & $-0,514$ & $0,002^{*}$ \\
& P2 & 0,275 & 0,110 \\
& P3 & 0,988 & $0,000^{*}$ \\
& P4 & 0,131 & 0,454 \\
\hline \multirow{2}{*}{ Disco Dosador B } & P1 & $-0,211$ & 0,253 \\
& P2 & $-0,135$ & 0,470 \\
& P3 & 0,985 & $0,000^{*}$ \\
& P4 & $-0,224$ & 0,225 \\
\hline
\end{tabular}


A alta correlação entre a pressão no ponto P3 e os valores de vazão média pode ser explicada considerando-se o teorema de Bernoulli: o total de energia do sistema no ponto P3 é resultante dos componentes elevação $(\mathrm{z})$, pressão $(\mathrm{p} / \gamma)$ e cinemática $\left(\mathrm{v}^{2} 2 \mathrm{~g}^{-1}\right)$.

Embora os valores de pressão encontrados nos pontos do circuito hidráulico e pneumático sejam específicos do projeto da máquina avaliada neste trabalho, a comparação com os valores encontrados no trabalho de JOLLANDS (1991) mostra que os valores de pressão são baixos, menores que $12 \mathrm{kPa}$. $\mathrm{O}$ volume de ar produzido pelo ventilador radial e conduzido até o reservatório de calda é reduzido e insuficiente para gerar grandes pressões, pois grande parte do volume de ar é direcionado para o interior do ducto aplicador criando a corrente de ar.

\section{CONCLUSÕES}

Os resultados obtidos com água permitem concluir que as vazões são mais uniformes no intervalo de aplicação entre $-60^{\circ}$ e $-20^{\circ}$, utilizando o disco dosador B.

Nos intervalos de aplicações para ângulos positivos, que na prática é a maioria dos casos, o disco dosador B apresenta melhor uniformidade.

\section{REFERÊNCIAS}

AMERICAN SOCIETY OF AGRICULTURAL ENGINEERS. Terminology and definitions for agricultural chemical applications. St. Joseph, 1996. p.159-61. (ASAE S 327.2).

ASSOCIAÇÃO BRASILEIRA DE NORMAS TÉCNICAS. TB-344: aplicação de defensivos agrícolas. Rio de Janeiro, 1988. 4 p.

ASSOCIAÇÃO BRASILEIRA DE NORMAS TÉCNICAS. NBR 12936: pulverizador agrícola. Rio de Janeiro, 1993. 5 p.

CENTRO DE VIGILÂNCIA EPIDEMIOLÓGICA. DIVISÃO DE ZOONOSES. Disponível em: <http:// www.cve.saude.sp.gov.br/htm/Den_dir.htm>. Acesso em: 18 set. 2001.

FUNDAÇÃO NACIONAL DE SAÚDE. Boletim eletrônico epidemiológico. Disponível em: <http// www.funasa.gov.br/pub/boletim_eletronico/epi/boletim/eletronico/epi_\%200101.pdf>. Acesso em: 19 set. 2001.

FUNDAÇÃO INSTITUTO BRASILEIRO DE GEOGRAFIA E ESTATÍSTICA. Censo demográfico 2000. Disponível em: <http://

www.ibge.gov.br/ibge/estatistica/populacao/censo2000/sinopsehp?tipo=21\&uf=00>. Acesso em: 17 set. 2001.

JOLLANDS, P. Evaluation of knapsack mistblowers for the control of coffee leaf rust in Papua New Guinea. BCPC Monograph, Duns, v.46, n.1, p.177-84, 1991. 